\title{
EVIDENCES OF EPISODIC MASS ACCRETION IN LOW-LUMINOSITY EMBEDDED PROTOSTARS
}

\author{
Hyo Jeong Kim ${ }^{1}$, Neal J. Evans II $^{1}$, Michael M. Dunham ${ }^{2}$, \\ JeOng-Eun LeE ${ }^{3}$, And Klaus M. POnTOPPIDAN ${ }^{4}$ \\ ${ }^{1}$ Department of Astronomy, The University of Texas at Austin, 1 University Station, C1400, Austin, Texas 78712 \\ ${ }^{2}$ Department of Astronomy, Yale University, P.O. Box 208101, New Haven, CT 06520, USA \\ ${ }^{3}$ Department of Astronomy and Space Science, Kyung Hee University, Yongin-si, Gyeonggi-do 446-701, Korea. \\ ${ }^{4}$ Space Telescope Science Institute, Baltimore, MD 21218, USA \\ E-mail: hyojeong@astro.as.utexas.edu \\ (Received July 01, 2012; Accepted July 26, 2012)
}

\begin{abstract}
We present Spitzer IRS spectroscopy of $\mathrm{CO}_{2}$ ice toward 19 young stellar objects (YSOs) with luminosity lower than $1 \mathrm{~L}_{\odot}$. Pure $\mathrm{CO}_{2}$ ice forms only at elevated temperatures, $T>20 \mathrm{~K}$, and thus at higher luminosities. Current internal luminosities of YSOs with $L<1 \mathrm{~L}_{\odot}$ do not provide such conditions out to radii of typical envelopes. Significant amounts of pure $\mathrm{CO}_{2}$ ice would signify a higher past luminosity. We analyze $15.2 \mu \mathrm{m} \mathrm{CO}_{2}$ ice bending mode absorption lines in comparison to the laboratory data. We decompose pure $\mathrm{CO}_{2}$ ice from 12 out of 19 young low luminosity sources. The presence of the pure $\mathrm{CO}_{2}$ ice component indicates high dust temperature and hence high luminosity in the past. The sum of all the ice components (total $\mathrm{CO}_{2}$ ice amount) can be explained by a long period of low luminosity stage between episodic accretion bursts as predicted in an episodic accretion scenario. Chemical modeling shows that the episodic accretion scenario explains the observed total $\mathrm{CO}_{2}$ ice amount best.
\end{abstract}

Key words: star formation; Spitzer IRS observation; conferences: proceedings

\section{INTRODUCTION}

The standard model explains that star formation is a continuous process (Shu et al., 1977). However, recent studies indicate that star formation does not occur continuously (Evans et al., 2009; Dunham et al., 2008). One possible scenario of non-continuous accretion models is the episodic accretion. If we can find imprints of past high luminosity/temperature at low luminosity YSOs, it can be evidence of episodic accretion.

In the interstellar dust, pure $\mathrm{CO}_{2}$ ice formation requires at least $20 \mathrm{~K}$. Dust temperature at an envelope of a YSO with $L_{i n t}=0.7 \mathrm{~L}_{\odot}$ is lower than $20 \mathrm{~K}$, which is too low to form pure $\mathrm{CO}_{2}$ ice. If mass accretion occurs episodically, low luminosity YSOs can form pure $\mathrm{CO}_{2}$ ice during burst stages, which does not disappear during quiescent stages. We tested this idea with Spitzer IRS Short-High mode observation and the chemical evolution modeling.

\section{OBSERVATIONS, ANALYSIS AND MODEL- ING}

We detected the $15.2 \mu \mathrm{m} \mathrm{CO}_{2}$ ice absorption line in all 19 sources. The observed flux is converted to optical depth, and fitted using 5 components of laboratory data (three different kinds of $\mathrm{CO}-\mathrm{CO}_{2}$ mixtures, pure $\mathrm{CO}_{2}$ ice, water-rich $\mathrm{CO}_{2}$; Pontoppidan et al., 2008). Then each optical depth is integrated to get the column density, N (see Figure 1).

The fraction of pure $\mathrm{CO}_{2}$ ice is plotted against luminosity in Figure 2. YSOs with $L>1 \mathrm{~L}_{\odot}$ have almost 

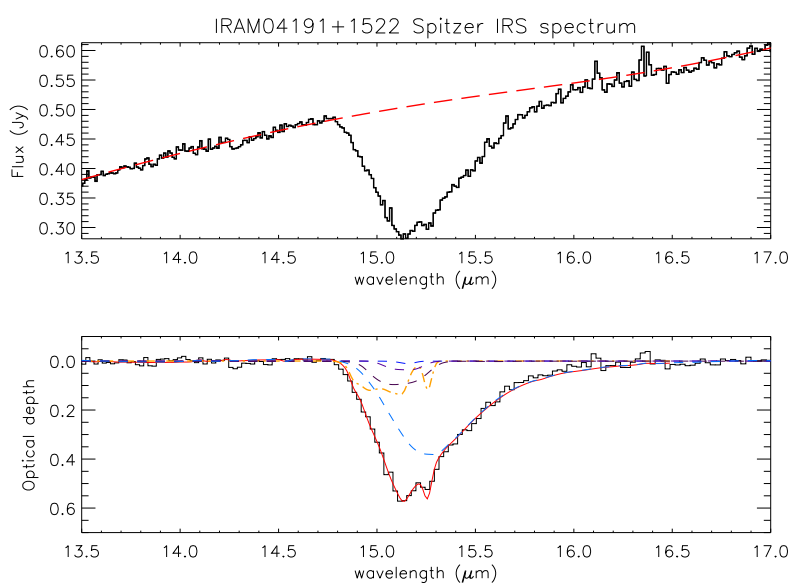

Fig. 1. A sample of Spitzer IRS spectrum of $\mathrm{CO}_{2}$ ice absorption line and the laboratory data fit. The source is IRAM $04191+1522$, a very low luminosity object, which has internal luminosity $0.08 \mathrm{~L} \odot$. Upper: Observed flux (black solid) and best fit continuum (red dashed). Lower: Optical depth(black solid), sum of all the ice components (red solid, best fit model), pure $\mathrm{CO}_{2}$ (yellow dash-dot), $\mathrm{H}_{2} \mathrm{O}$-rich $\mathrm{CO}_{2}$ ice (blue dashed), $\mathrm{CO}-\mathrm{CO}_{2}$ mixtures (purple dashed). Pure $\mathrm{CO}_{2}$ ice double peak is observed in the source.

the same ratio as low luminosity YSOs. The fraction of pure $\mathrm{CO}_{2}$ ice does not depend strongly on the luminosity. This supports that the currently existing central star is not the only heating mechanism of the envelope.

Using a chemo-dynamical model (Lee et al., 2004; Kim et al., 2011), we relate the amount of observed total $\mathrm{CO}_{2}$ ice, including that in mixed ices, to the accretion scenario. We investigate 4 scenarios, continuous and episodic accretion with and without $\mathrm{CO}$ ice to $\mathrm{CO}_{2}$ ice conversion. The scenario with episodic accretion and $\mathrm{CO}$ ice to $\mathrm{CO}_{2}$ ice conversion explains the observed range of $\mathrm{N}$ and the luminosity spread of YSOs best.

\section{ACKNOWLEDGEMENTS}

This work is supported by the NASA under RSA 137730 issued by the JPL. H. J. K and N. J. E. acknowledge support from NSF grant AST-0607793. J. E. L. was supported by Basic Science Research Program through the NRF of Korea (No. 2011-0004781).
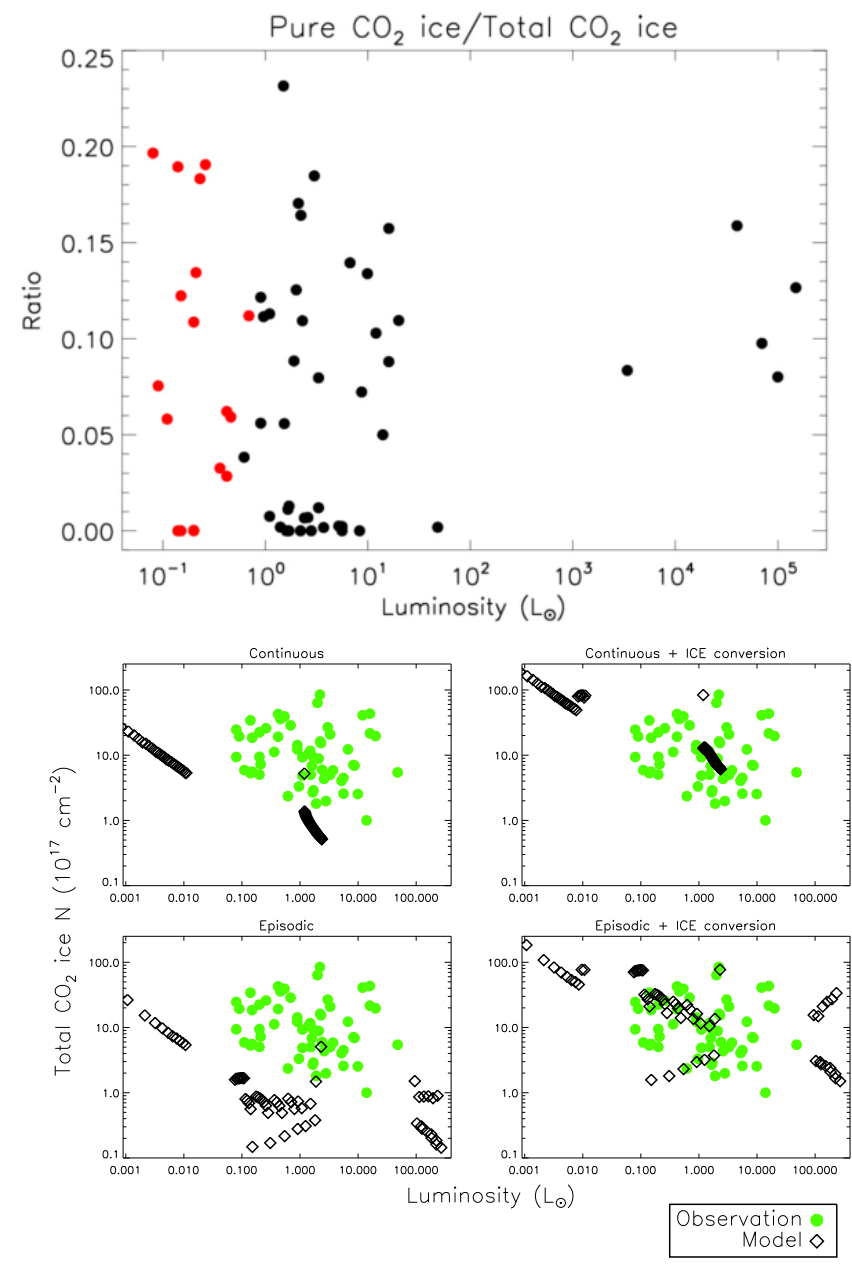

Fig. 2. Upper: The ratio of pure and total $\mathrm{CO}_{2}$ ice vs luminosity. Red dots are from this work and black dots are from Pontoppidan et al. (2008). Lower: Total $\mathrm{CO}_{2}$ ice $\mathrm{N}$ versus luminosity obtained from the chemical model and the observations (Kim et al., 2012).

\section{REFERENCES}

Dunham, M. M., et al., 2008, Identifying the LowLuminosity Population of Embedded Protostars in the c2d Observations of Clouds and Cores, ApJS, 179, 249

Evans, N. J., et al., 2009, The Spitzer c2d Legacy Results: Star-Formation Rates and Efficiencies; Evolution and Lifetimes, ApJs, 181, 321

Kim, H. J., et al., 2011, The Spitzer c2d Survey of Nearby Dense Cores. XI. Infrared and Submillimeter Observations of CB130, ApJ, 729, 84

Kim, H. J., et al., 2012, $\mathrm{CO}_{2}$ Ice Toward LowLuminosity, Embedded Protostars: Evidence for Episodic Mass Accretion via Chemical History, Submitted 
Lee, J. -E., Bergin, E. A., \& Evans, II, N. J., 2004, Evolution of Chemistry and Molecular Line Profiles during Protostellar Collapse, ApJ, 617, 360 Pontoppidan, K. M., et al., 2008, The c2d Spitzer Spectroscopic Survey of Ices around Low-Mass Young Stellar Objects. II. $\mathrm{CO}_{2}$ ApJ, 678, 1005

Shu, F. H., 1977, Self-similar Collapse of Isothermal Spheres and Star Formation, ApJ, 214, 488 